\title{
Shifts Between Search Stages During Task-performance in Mediated Information Seeking Interaction
}

\author{
Judit Olah \\ Queens College, Graduate School of Library and Information Studies, Flushing, Queens, NY. judit_santon@qc.edu.
}

The research reported here focuses on the users' information seeking behavior. Set in the framework of the interactive information retrieval paradigm, the research examines the micro-dynamics of the information seeking process, and explores search stages that users pursue as they proceed to solve their information problem. The research analyzes the users' information seeking behavior as they move between search stages, and looks for patterns in the transition. The observed shifting behavior allows for examining the question of predictability. The observed shifting patterns lead to the development of a new model for information seeking behavior that describes multiple search stages, aspects and dimensions. The model represents a non-linear process of multiple re-iterative cycles that make up the overall flow of communication with the information system. The model expands significantly earlier theoretical and empirical models as it represents a series of re-iterative processes that characterize the information seeking interaction.

Introduction

The paradigm shift in information retrieval research marked the understanding of the dynamic nature of the interaction; at the levels of relevance, cognition and interaction (Robertson, Hancock-Beaulieu, 1992). The interactive model shows a dynamically developing information need that is the product but also the generating force behind the interaction; information systems research advanced to replace the traditional systems with highly responsive and interactive information systems. User modeling has grown into an important area of investigation within the interactive paradigm, resulting in numerous theoretical and to a lesser degree empirical 
models. As we advance in our understanding of the nature and the patterns of the user's information seeking behavior we create recommendations for more intelligent information systems that can assist the users in the act of formulating their query but also in clarifying, focusing their information needs. As Belkin, et al. had stated in 1995, it is from a deep understanding of the nature of the interaction that we can move to outline specification of a system design that can support and enhance the user's communication with the information system (Belkin, et al. 1995).

\section{Research Problem}

The research focuses on the users' problem solving behavior, and it aims to map out the patterns of interactive processes as they contribute to the formulation and conceptualization of the user's information need. User behavior modeling focuses on the user's capabilities of understanding the system feedback, and understanding his options, offered by the system, to manipulate his original information problem, and present it to the system.

The research identifies typical search stages as the interaction progresses, and looks for transition patterns between those stages via the analysis of the user's information seeking behavior. In particular, the research focuses on the transition between the search stages and explores if shifting between stages can be connected to degrees of predictability. Via understanding the transition patterns we may advise a more effective mediation of the IR processes, regardless whether the mediator is a human being or the information system.

Deriving from this goal the following research questions were presented:

1. What stages do the users go through during their information seeking interaction?

2. What sequence can be observed between those stages?

3. Can we predict user behavior based the observed sequences?

The detailed analysis of the user's behavior, and in particular, looking for patterns in this behavior, can enhance the design of information systems, as well as it contributes to the advancement of interface design. 


\section{Theoretical Framework}

Within the larger theoretical area of interactive information retrieval research, two areas are of special interest to this study: 1. modeling the user's information problem; 2 . modeling the user's problem solving behavior. This latter represents the immediate theoretical foundation to the research presented here.

The interest in the micro-dynamics of the search process is not a new phenomenon. Research in the area of problem solving behavior resulted in theoretical and empirical models either addressing the characteristics of the problem solving process; or analyzing the patterns of the problem solving behavior. Particularly the early models were fairly general in addressing information seeking/ retrieving at a macro level; the more recent models have become increasingly fine-tuned in either describing characteristics of problem solving or the patterns of the problem solving processes; namely models developed by Denin (Denvin, 1986), Bates (Bates, 1989), Spink (Spink, 1997).

Interest in search stages reaches back to Ellis (Ellis, 1989), this type of research has become robust with Kuhlthau's contribution (Kuhlthau, 1991) on defining search stages, and was further refined by Belkin, focusing on characteristics of search strategies (Belkin, et al 1995) and Xie (Xie, 2000). Xie's work also signifies a deepening interest in obsenving shifting patterns between search stages, and lead to works by Robins (Robins, 2000), and Bhavnani (Bhavnani, 2001).

\section{The design of the study}

\section{Dataset}

The data set was randomly selected from a larger pool of data, originally collected in 1999 during a joint research conducted by the University of North Texas and the University of Sheffield, UK (Spink et al., 2002, Wilson, et al., 2002). 38 users were randomly selected from the total of 87 users were observed at the University of North Texas in information seeking interaction. Demographic 
Table 1. Demographic information

\begin{tabular}{|l|l|l|l|}
\hline & Gender & $\begin{array}{l}\text { Average } \\
\text { age }\end{array}$ & $\begin{array}{l}\text { Degree } \\
\text { (grad) }\end{array}$ \\
\hline Females & $14(36,8 \%)$ & 39.1 & $85.7 \%$ \\
\hline Males & $15(39.5 \%)$ & 40.8 & $86.7 \%$ \\
\hline Unknown & $9(23.7 \%)$ & $\begin{array}{l}\text { Missing } \\
12\end{array}$ & $\begin{array}{l}\text { Missing } \\
11\end{array}$ \\
\hline
\end{tabular}

\section{Methodology for Data Collection}

The users were assisted by three experienced intermediaries while searching the DIALOG system. The users were volunteers recruited from university students, academics and non-academic employees at the campus. The users were requested to search a topic of their own interest. 


\begin{tabular}{|l|l|}
\hline Topical area & Users \\
\hline Science & 1 \\
\hline Medicine & 4 \\
\hline Humanities & 2 \\
\hline Social sciences & 24 \\
\hline Business administration & 1 \\
\hline Law & 6 \\
\hline
\end{tabular}

The interactions were captured in multiple ways:

1. Audio recordings of the user - -intermediary interaction.

Included in the full session were the operational steps of database selection as well as actions and cognitive steps taken in formulating the query. As the user and the intermediary worked jointly towards the information problem, they discussed concepts, ideas, steps, as well as commented on the displayed result set.

The discourse also included elements of discussion focusing on the test experiment: comments regarding the questionnaires, interpretations of various questions, the mechanics of carrying out the experiment were also recorded.

Finally, there is a layer of the social discourse captured, incidental communication; the participants commenting on various outside issues.

2. Computer search logs.

The search logs of each interaction was captured and saved. The search logs recorded the databases selected for the 
searches, the queries created by the joint efforts of the user and the intermediary, as well as the result set displayed for each query along with some of the technical difficulties experienced during the online search. The search logs included all operational moves, including errors, misspells, repeated runs of queries, etc.

3. Pre- and post-search questionnaires.

Users were asked to fill out an extensive questionnaire prior to the test, and a second one, after completing the search. The presearch questionnaire included questions about the user's search interest, the depth and scope of their knowledge of the subject area, as well as the user's prior exposure to the topic. The post-search questionnaire investigated the overall sense of change in the user's knowledge of the topic at hand. While the questionnaire was not directly applicable to the focus of this research, their existence is noted here, as they frequently appeared in the discourse. (A sample questionnaire to be demonstrated.)

\section{Methodology for Data Analysis}

The research shares the methodological foundations of earlier studies in that the discourse between the user and the intermediary provides clues to the mental model of the information problem mutually held by the participants of the interaction, and that the discourse uncovers important cognitive processes surrounding the information seeking actions and decision making processes. The focus of the discourse between the user and the intermediary reflects the knowledge shared by them, while it also provides cognitive evidence surrounding the information seeking actions.

The data analysis involved several major steps:

1. Discourse analysis of transcribed audio records

2. Definition of search stage in the context of this research

3. Development of a coding scheme

4. Coding the transcribed discourse 


\section{Creating coded strings of the discourse}

6. Observance of patterns of code occurrences.

The audio records have been fully transcribed and their contents were coded using the principles of discourse analysis (Brown, Yule, 1980). The discourse analysis suggested by Yule and Brown applies a functional analysis of content bearing phrases rather than a strictly linguistic approach. The results of the functional analysis were captured in a log indicating whether the utterances came from the user (U) or from the intermediary (I).

The search logs provided additional data, as they captured the users' queries and the retrieved data sets they interacted with. In the current study, reliability is established via the analysis of the search logs that not only helped to interpret the contents of the discourse, but captured the query formulations thus provided coding reliability.

\section{Validity/Reliability}

Discourse is situational in nature, consequently, the validity of the methodology is situational as well. The meaning of the discourse is interpretative and relational, and the interpretation is only possible within a known context. The strength of internal validity here comes from explanation validity that is the acceptability of the explanation, and translation fidelity, meaning the grounding of the theory in data. Thus ample examples are to be provided to demonstrate instances identified in the course of the study. External validity also depends heavily on conceptual evidence. But the rich and detailed illustrations allow the audience to 'try the examples' to see whether they fit their experience (Krathwohl, 1993). As the discourse is coded within a known context (the structured lab experiment, known retrieval system (DIALOG), known retrieval technique, and using a relatively limited vocabulary, the interpretations are less ambiguous and more valid. Within the framework of the qualitative research, the researcher is looking for commonalities. Validity is also provided as explanation validity, where the codes established are grounded in the ample presentation of examples.

Coding reliability was established via observation triangulation by the combined analysis of discourse and search logs. The reliability of interpreting the discourse correctly and consequently, establishing search stages accurately, relates back to the lessened degree of ambiguity within the parameters of the research discussed above. 


\section{Limitations}

Some of the study's limitations originated from the fact that this is a laboratory study, which, with its artificial environment and intermediary interaction limits the reality of the experience. The laboratory circumstances on the other hand, allow for high level of observability. A huge advantage of the study is that the users were experimenting with a topic of their own interest. Limitations also came from the fact that this is a qualitative study with qualitative measures and methods which limits the degree of conclusions.

\section{Results}

The Definition of the Search Stage

Table 3. Fourteen identified search stages 


\begin{tabular}{|c|l|}
\hline Code & Name of Search Stage \\
\hline 1 & Search intentions \\
\hline 2 & Prior Experience \\
\hline 3 & Database Selection \\
\hline 4 & Query Formulation \\
\hline 5 & Desirable format/size \\
\hline 6 & Review/Evaluate Result Set \\
\hline 7 & Review Search Progress \\
\hline 8 & Physical Delivery of Result Set \\
\hline 9 & Discussion of Search Skills \\
\hline 10 & Social Chat \\
\hline 11 & Technical/Computer Problems \\
\hline 0 & Exit System/End Task \\
\hline A & Administration of Experiment \\
\hline B & Scheduling of Experiment \\
\hline
\end{tabular}


For the purposes of this study a search "stage" is identified upon two components: the topic of discourse as it reflected in the search activities that are being carried out, and the analysis of search logs, when it was applicable. A search stage then is reflected in the topic of the discourse and it is grounded in the operational steps of conducting the search, regardless if those are carried out by the user or by the intermediary. A search stage is composed of cognitive and operational elements. It is identified as a unit in discourse and confirmed, when possible, by the strategic steps of information retrieval as those are captured in the search logs. The elements are not interchangeable. The cognitive and strategic / operational elements are typical to a single stage only and to no other.

A search stage incorporates cognitive and strategic elements that are typical only to that particular stage: e. g. the stages of Database Selection, Query Formulation, Review Results and Technical/ Computer Problems. While the principle sources of analysis were the transcribed discourses, the search logs critically underpinned the discourse, showing actions actually taken.

Transition from one stage to another is identified through changes in the vocabulary of the utterances, and confirmed, when appropriate, by the analysis of the search logs.

The sequence of various stages solely reflects a sequence in time, and not a cognitively hierarchical sequence (unlike Kuhlthau's search stages).

A transition or shift signifies a transition from one stage to another. Shifts can be initiated by either participant. Some of the shifts were clearly demarcated by the turn-taking behavior between the user and the intermediary. Less typically, shifts were initiated within the discourse of either participant. Both types of shifts were identified through the changes in the vocabulary and the interactions reflected in the search logs.

\section{Transition Patterns between Search Stages}

Individual information seeking interactions were coded in an alphanumerical code to reflect the moves between search stages, and 
Table 4. Sample alphanumerical string

\section{A-3-10-1-2-1-2-1-3-10-1-4-6-4-6-1-8-4-10-6-8-6-1-7-8-11-10-A}

38 such strings were created for the 38 users showing:

1. The sequence of search stages in the interaction;

2. The number of search stages pursued in the interaction.

Users proceeded through various number of search stages, resulting in various lengths of coded strings. The lengths of interactions were not measured, thus longer strings did not reflect longer interactions, only a higher number of search stages pursued. The alphanumerical strings were tabulated for 38 users, reflecting search stages pursued by users as their first, second, third, etc. Based on observed sequences, a transition probability was calculated, showing the likelihood of proceeding to a particular stage after a certain search stage.

The tabulation of the coded interactions resulted in two observations:

1. Transition frequency.

2. Transition probabilities between search stages.

\section{Patterns of Shifting Between Search Stages}


Shifting occurred in two patterns:

1. In straightforward proceeding to a next different stage, in a linear pattern;

2. Shifting back to, re-iterating, a previous stage.

Re-iterative shifts were observed between several search stages.

All search stage transitions are to be illustrated with samples from the transcribed discourse.

\section{Concept of Sequential Dependencies}

The transition matrixes enlisted the probabilities of shifting from a current search stage to another one. One of the research objectives was to explore the degrees of predictability involved in the information seeking behavior. For this, the Markov-model seemed to offer an appropriate solution.

The Markov-model was developed in the mid-1970s to describe a probabilistic rule between events that are conditioned by the most recent experiences of the user (Cooper, Chen, 2002). The model states that the user's behavior can be classified into a relatively small number of well-defined groups called states. The model makes a prediction regarding the user's next most likely state based in prior states. First-, second- and third order models were identified based on the length of the strings.

The observed shifts of proceeding from one search stage to the next, are fundamentally, first-order Markov models. The prediction is limited, as it only claims that the observed search stage enables us to make predictions regarding the user's shift onto the next one.

First-order models, observed in this research, allowed for three important observations:

1. The existence of re-iterative loops;

2. High probability of shifting outside stages 4 and 6 ; as noted $62.5 \%$ of total shifts occurred outside stages 4 and 6 ; 
3. The appearance of stages 2,7 and 8 in the models with high transitions probabilities.

Keeping in mind the limited predicting power of the third-order Markov-chains, and also the fact that the scope of this research based on a qualitative analysis of information seeking behavior, the conclusions regarding third-order models will be limited to the fact, that they occurred in the interactions in two patterns: looping and directional chains were observed.

\section{Discussion}

The analysis traced the sequence of occurrences of various search stages and examined the order of those. By doing so, the research investigated the patterns of shifting between search stages by simply tabulating the frequency of transition from a search stage to another one, regardless if the shift occurred in the beginning, middle, or final stages of the interaction. 


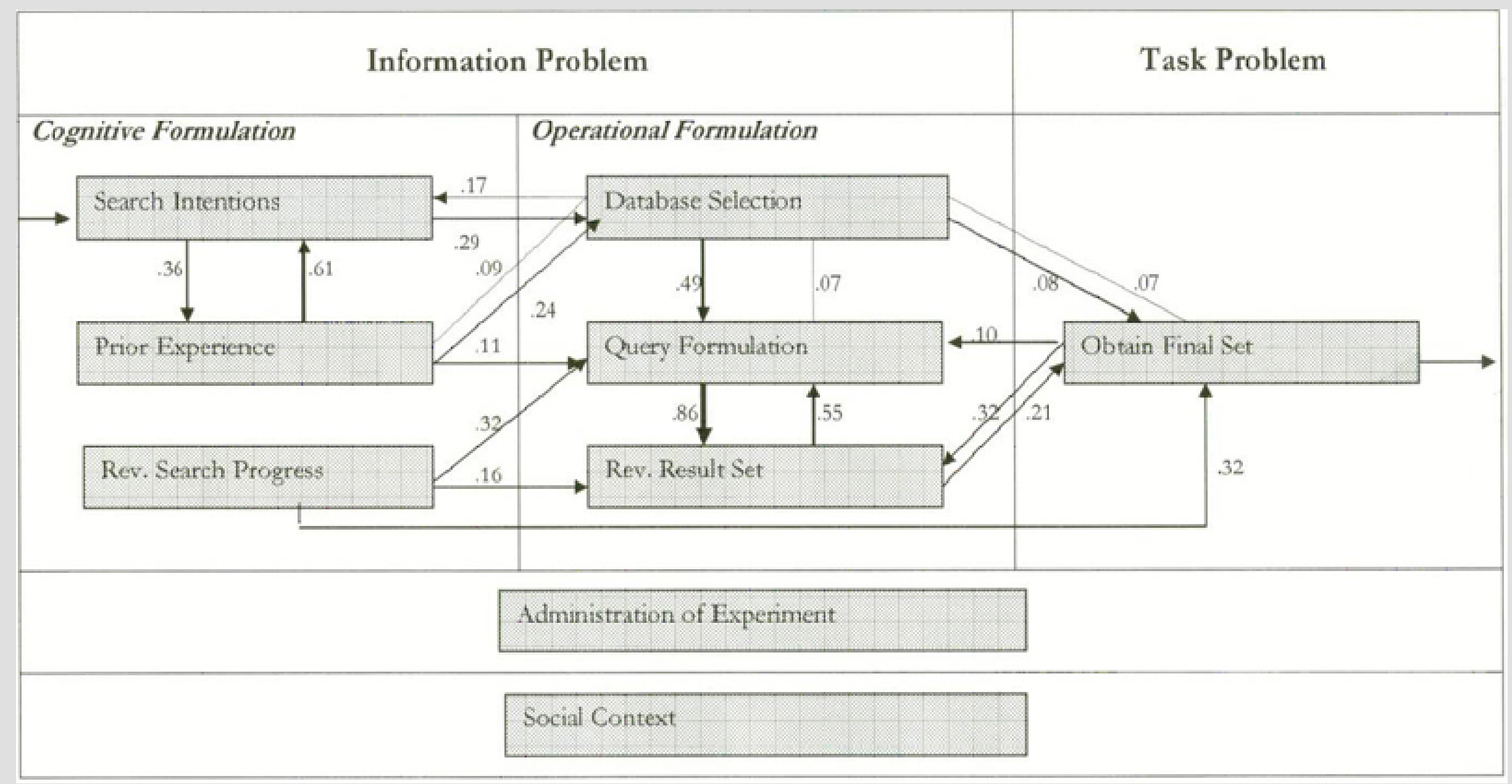

Figure 1: The shifting patterns

The model in Figure 1 represents the shifting patterns with first-order sequential dependencies; it is a graphical representation of the highest probabilities observed. The transitions show the probabilities after eliminating the stages relevant to the administration of the experience (Stages A and B). Those stages appear in the model as contextual elements.

Three important obsenvations were made regarding the progress of the interaction: 
1. Patterns of shifts

The overall progress of the interaction is built on a series of re-iterative loops, repeating certain stages before proceeding to the next one.

2. Extent of shifts

The reiteration is limited, as the user tends to repeat one stage before proceeding to the next one.

3. Critical points of multiple shifts in the interaction.

Three such critical points were identified: Database Selection, Review Result Set, and Physical Delivery Of Result Set.

The graphical representation of the model also helps to identify groups of search stages that are similar in character. Three search stages involve cognitive activities only, i. e. Cognitive Formulation: Search Intentions, Prior Experience, and Review Search Progress; while others involve both cognitive and operational activities, i.e. Operational Formulation: Database Selection, Query Formulation, and Review Result Set. The transition patterns of the information seeking interaction is constituted by several loops and linear moves forward, reflecting shifts between the cognitive and operational plains of the interaction.

Further the user's cognitive occupation with the task goal, as to obtain, have, copy, e-mail, the final set of documents, appears very early on in the interaction, and remains continuously important throughout the interaction.

\section{Theoretical Implications}

The research focused on the transition between search stages that were pursued during information seeking interaction. The analysis of transition patterns lead to the exploration of degree of predictability in the information seeking behavior. A new model of shifting behavior between search stages was designed, based on the observed first-order sequential dependencies. The model shows the 


\section{The concept of Search Stages}

A total of fourteen search stages were identified, twelve of those related directly to the test administration, including discussion of filling out the questionnaires, and timing/ scheduling the test experiments.

How do these stages relate to research findings of other authors? While Taylor focused on the changes in cognitive hierarchies resulting in the user's compromised need as presented to the system, he also realized that the user's initial inquiry presented to the system is merely a micro-event in a shifting non-linear adaptive mechanism (Taylor, 1968). Ellis's cognitive behavioral elements were later developed into cognitive search stages by Kuhlthau (Kuhlthau, 1991), Wilson (Wilson, 1999), and Vakkari (Vakkari, 2001).

Several authors examined shifting patterns between search stages as Daniels et al (1985), Spink (1997), Xie (2000), Robins (2000), Bhavnani (2001). These authors most typically approached the patterns of shifting as the user's response to the result set displayed by the system. In the study by Xie, shifts between intentions and strategies were defined by cognitive elements (Xie, 2000). Robins focused on shifts between cognitive components.

The empirical model presented here is theoretically closest to the strata model (Saracevic, 1996). That model suggested various interactions occurring at the surface, at the situational, and at the cognitive levels, stating that the user moves between spheres as the interaction progresses. Shifting between spheres included interactions with layers of engineering, content and processing levels, and the expectation that the user understands the pragmatic implications of processing and some of engineering as he navigates through these layers. Actions are iterative, concurrent and cohesive, rather than sequential, atomic and independent (Saracevic, 1996).

\section{Empirical Evidence for Shifting Behavior}


The empirical results presented in this research brought results in the following critical areas:

First of all, strong shifting patterns were found between search stages outside query formulation and reviewing the result set. The research results presented here extended the scope of the user's shifting behavior observed by these earlier studies. In light of the results, we recognize that the most important and the highest probability transitions occur between query formulation and reviewing the result set displayed for a query, but the research also gathered empirical evidence that a significant proportion of shifting occurred between other search stages. More than $50 \%$ of the total transitions occurred between other search stages. Shifting between other search stages, was not prompted by a sense of dissatisfaction with the displayed results, but more active steps of cognitive and operational formulation, ultimately moving towards the task goal. Thus, the interaction was not only a re-action to the system's response delivered as displayed information records, but also in the user's cognitive and operational strategies actively pursued in the dynamic communication with the information system.

Re-iteration was far more extended than traditionally assumed. Fundamentally, the dynamic dialog between the user and the information system is constituted by a series of re-iterative loops, where search stages are often repeated before a forward move is pursued. The observed second- and third-order Markov models confirmed multiple re-iterations of previous search stages before a forward transition was made.

The empirical model presented by this research shows the user shifting between cognitive and operational aspects of the interaction, that include not only cognitive transitions, but also the mechanics of database selection, query formulation, and reviewing the results.

As the model shows, shifting occurs between multiples plains of the information seeking interaction that will lead to our next discussion on shifting patterns.

\section{Patterns of Shifting}

Shifting Between Various Aspects of the Interaction 
picking model, or the Spink-model (Spink, 1997) that looked at the impact of system output prompting re-iterative feedback loops.

As our model shows, shifts occur at several levels of the interaction, thus the empirical data gathered by this research supports the theoretical model of Saracevic (Saracevic, 1996). The users shift between stages within a single aspect of the interaction, cognitive or operational, shift between the aspects, and shift between the dimensions of the interaction as well.

The critical extension of shifting behavior in our model is addressed two areas:

1. The role of system feedback in prompting further query formulation;

2. The cyclical re-looping to certain search stages beyond query formulation.

Earlier studies stated that shifting is generated by two measures: relevance and uncertainty;

- Relevance regarding the user's judgment about the displayed result set, that, if unsatisfactory, prompts the user to repeat the query formulation stage;

- Uncertainty that, if unresolved, will prompt the user to start the interaction all over again.

While we have shown direct evidence for relevance based transitions, as the highest probability shifts occurred between query formulation and reviewing the result set displayed for the query, there was no direct evidence gathered how these shifts would be generated by lessening or increasing uncertainty. On the other hand, we observed shifts generated by cognitive centering, or recentering, either to shifting to reflection, or to focusing on the task objectives of the interaction. In our model, the cyclical relooping behavior was found at multiple levels, and only one of those occurred at the stage of query formulation.

\section{Length of Transition Patterns}

The observed re-iterative cycles are short in terms of the number of search stages involved. Time was not measured in this study, thus we cannot make a statement if these loops were pursued in quick re-iterations, we saw, however, that usually the user shifted one 
stage back before he chose to move forward. This statement is supported both by the existence of the observed first-order sequential dependencies, and also by the fact, that even the second- and third-order dependencies involved two search stages only with multiple reiterations between them. Theoretically, these observations are close to Spink's result (Spink, 2000) of short Web-search interactions and also to Spink's feedback model that showed short re-iterations.

Empirical evidence did not support the "start all over" concept represented by for example the Wilson (Wilson, 1999) modification of the Kuhlthau (Kuhlthau, 1991) model. Wilson suggested considering to adopt Kuhlthau's search stages within a single interaction, where the user's moves are propelled by series of uncertainty loops, suggesting that user starts the information seeking 'all over' once a point of uncertainty has been reached. It was rare that a user in our research shifted to Stage 1 . in the later stages of the interaction.

\section{The Role of Task Objectives}

Both theoreticians, as well as recent empirical investigations, have emphasized that the role of the user's sense of his task goal has not been adequately appreciated. Recently, Vakkari (Vakkari 1999, 2001), Borlund (Borlund, 2000), and Ingwersen (Ingwersen, 2000) addressed the significance of the task objectives, in terms of its less dynamical nature, and its strongly defining role of how the user proceeds with the interaction. In fact, Ingwersen cognitive model already attributed a key significance to the work/ task dimension of the interaction. Looking back to earlier works, the Kuhlthau's model of cognitive and affective stages also included important finding regarding the user's perceptions of his task goal. Further, the basic tenet of the Saracevic model rests on the concept of fundamental goal of "acquisition - cognition - application," implying that it is not cognition but application that ultimately guides the course of the interaction.

In our model, shifts to the task objectives occur early on, and the frequency of shifting to this stage remained steady throughout the interaction.

\section{The Role of Reflection in Cognitive Formulation}


Reflection appears both in the early and in the later phases of the information seeking interaction although with a different purpose. In the early stages reflection typically appears as relating to the prior experience with the topic at hand. In this phase, reflection is a tool of clarification of the subject area and the topic itself, part of the general formulation, expression, and definition of one's search intentions.

In the later phases, reflection serves as a centering experience, to focus the interactive process, as "so far we have done", "so far we have checked", etc. cognitive processes assist the re-focusing of the search intentions, the clarification that what is happening is in deed in line with the information goal, and the task goal.

\section{Predictability in Shifting}

The research has presented evidence that some $63 \%$ of typical transitions occur outside the query formulation/ result set loop. Several first-order sequential dependencies have been identified. The second- and third-order sequential dependencies in this research basically confirmed the heavily cyclical nature of the interaction, confirming that the information seeking interaction is not a linear process but a series of cyclical loops of repeated steps, that do not go back most typical than one previous step. Note however, that the second- and third-order sequential dependencies had limited implications as their analysis was primarily qualitative and they were used to support to re-iterative pattern of behavior without drawing further conclusions about shifting patterns.

\section{Taxonomy of Search Stages}

Search stages were classified based on their characteristics. A taxonomy does not explain a phenomenon, but it helps to clarify how the concepts relate to each other in order to generate further research interest and theoretical investigation. Some of the search stages were characterized by strictly cognitive activities, while others involved both cognitive and operational activities. Three search 
stages then fell into cognitive formulation: Search Intentions, Prior Experience, and Review Search Progress. Three search stages fell into operational formulation: Database Selection, Query Formulation, and Review Result Set. The cognitive and operational formulations are two aspects of the information problem related dimension of the information retrieval interaction. This is a dynamic dimension of the interaction, characterized by not only the dynamic moves of the user between the various search stages, but also by the dynamic evolvement of the user's information needs, as it is reflected through repeated addressing of search intentions, renewed database selections, query formulations and re-formulations, etc. A more constant and less dynamic dimension of the interaction is related to the task object of the user that is related to very early on in the interaction, and remains an important concern of the user throughout the experience. The user's primary goal regarding the task objectives seems very similar with Kuhlthau's (1991) finding of 'gather' and 'complete' as objectives of the interaction.

\section{Information Seeking as Adaptive Mechanism}

Our results demonstrate that it is not only the parameters of the information system that forces the user to adapt his information need, but also the parameters, the objectives of his information task goal. The user explores, investigates the information resources and evaluates the presented information objects within the parameters of his task, and his judgment whether those objects will contribute to successfully solve his task problem.

Bringing the task goal into the investigation, and attributing a much bigger weight to its role in defining the progress of the interaction, emphasizes the user's information seeking interaction as an adaptive behavior. The task problem, being the objective representation of the user's subjective information problem that already has been compromised in the system's bibliographic language.

\section{Conclusions}

The research investigated patterns in the user's information seeking behavior with the purpose of exploring degrees of predictability 
in the interaction. Any degree of predictability would greatly assist information system design, as we devise increasingly dynamic information systems that act as true mediators of the information seeking process.

Twelve search stages were identified in the course this research that were directly related to the information seeking activity. The search stages formulate various aspects and dimensions of the information seeking process. By obsening the user's shifting behavior the research identified critical points in the interactive process. The information seeking communication flows towards solving the task problem; an objective, constant, less dynamic dimension of the user's information problem. As the communication progresses, the user completes loops, as he repeats search stages. Via the loops the user pursues various aspects and dimensions of the information seeking interaction.

The model of shifting patterns shows several reiterative loops. The shifts occur in a complex system of cognitive and operational planes. Instead of approaching the problem in a linear motion, the user is in constant transition between the cognitive and operational aspects of the interaction.

The research fits in the theoretical directions of earlier studies focusing on the user's problem solving behavior, it builds on their results, but it also extend those significantly while it contributes empirical results to the interactive IR research. It replaces the traditional concept of a linear information retrieval with a model of shifting patterns as the user pursues a series of reiterations during the course of the information interaction.

The model presented here fits into what Buckland presented as information-as-process, and demonstrates that certain characteristics coexists and pursued throughout the communicative process. What Saracevic envisioned as a variety of interactions at multiple levels, is represented here as multiple characteristics pursued parallel. Instead of a user moving along from phase to phase, as the early user behavior models had envisioned, or pursuing repetitions of episodes, here the user is pursuing cognitive, operational and task related characteristics of the overall information problem.

In terms of the pattern of shifting, several conclusions have been made: the user most typically re-iterates one previous search stage. The second- and third-order Markov models observed, showed, that the user was willing to reiterate a previous step multiple times. The length of a typical shift was short, even higher order models typically included two search stages only. 
The task objectives are of key significance to the user, and the task awareness is present and was observed throughout the entire interaction.

The role of reflection seems to be of high cognitive significance, as the user often addressed his prior experience and exposure to the problem area, and often regrouped during the course of the interaction via reflecting upon the progress.

The research questions addressed the concept of predictability. While the higher order models' implications are limited within the context of a qualitative study, they do indicate patterns of predictability of user behavior. Also, the model suggests critical points during the course of the interaction that can be extremely helpful for a system mediator.

The suggested taxonomy grouped search stages according to their cognitive or operational features and suggested that the information problem dimension and the task objective dimension of the information seeking interaction are equally critical elements of the interaction.

Consequently, the information seeking behavior of the user can be extended as an adaptive behavior reflected in a dynamically changing information need, and mediated by a more static, constant and objective task.

\section{Implications for Future Research}

With its empirical results, the research opened up new areas for future research to further explore the area of information seeking behavior modeling. There are several avenues to expand upon the investigation presented here: The research was designed with the involvement of intermediaries; and the research experiment may and should be repeated with end-user information seeking.

The analysis focused on the transition sequences and did not explore how long the user stayed in a certain search stage, or how swift the transitions occurred; a natural extension of the results will be the investigation of time in terms of the search stage transitions. 
The research experiment was designed in a highly structured bibliographic environment; it can be repeated in other structured bibliographic environment, such as the open public access catalogs, or take the experiment to less structured information systems as the World Wide Web.

\section{Acknowledgements}

The author sincerely thanks the invaluable suggestions and assistance of Tefko Saracevic, Nick Belkin, and Amanda Spink in the development of this research.

\section{References}

Bates, M. J. (1989). The design of browsing and berry picking techniques for the online search interface. Online Review, 13(5), 407-424.

Belkin, N.J., Cool, C., Stein, A., Thiel, U. (1995). Cases, scripts, and information-seeking strategies: on the design of interactive information retrieval systems. Expert Systems with Applications, 9(3), 379-395.

Bhavnani, S. K. et al (2001). Towards a unified framework of IR tasks and strategies. Proceedings of the Annual Meeting of the American Society for Information Science, 340-354..

Brown, G., Yule, G (1983). Discourse Analysis. Cambridge, UK: Cambridge University Press.

Borlund P. (2000). Experimental components for the evaluation of interactive information retrieval systems. Journal of Documentation, 56(3) 225-250.

Cooper, M.D., Chen, H.-M. (2002). Stochastic modeling of usage patterns in a web-based information system. Journal of the American Society for Information Science, 53(7), 536-548. 
Daniels, P.J., Brookes, H.M., Belkin, N.J. (1985). Using problem structures for driving human-computer dialogs. RIAO-85. Actes: Recherche de'Informations Assistee par Ordinateur, pp. 645-660. Grenoble, France: IMAG.

Dervin, B., Nilan, M. (1986). Information needs and uses. Annual Review of Information Science and Technology. Martha E. Williams, Ed. Vol. 21. pp. 3-33. Medford, NJ: Knowledge Industry Publications, Inc.

Ellis, D. (1989). A behavioral approach to information retrieval system design. The Journal of Documentation, 45(3), 171-212.

Ingwersen, P.(2000). Cognitive information retrieval. Annual Review of Information Science and Technology. Martha E. Williams, edr. Vol. 34, pp 3-51. Medford, NJ: Information Today, Inc.

Krathwohl, D.R. (1993). Methods of Education and Social Science Research: an integrated approach. New York: Longman.

Kuhlthau, C. (1991). Inside the search process: information seeking from the user's perspective. Journal of the American Society for Information Science, 42(5), 361-371.

Robertson, S.E., Hancock-Beaulieu, M.M. (1992). On the evaluation of IR systems. Information Processing and Management, 28(4), 457-466.

Robins, D. (2000). Shifts of focus on various aspects of user information problems during interactive information retrieval. Journal of the American Society for Information Science, 42(5), 361-371.

Saracevic, T. (1996). Interactive models in information retrieval (IR). A review and proposal. Proceedings of the 59th Annual Meeting of the American Society for Information Science, 33, 3-9.

Spink, A. (1997). Study of interactive feedback during mediated information retrieval. Journal of the American Society for Information Science, 48(5), 382-394.

Spink, A., Jansen, B.J., Saracevic, T. (2000). Real life, real users, and real needs: a study and analysis of user queries on the web. Information Processing Management, 36, 207-227. 
Spink, A., Wilson, T.D. Ford, N., Foster, A. Ellis, D. (2002). Information seeking and mediated searching. Part I. Theoretical framework and research design. Journal of the American Society for Information Science, 53(9), 695-703.

Taylor, R. S. (1968). Question-negotiation and information seeking in libraries. College and Research Libraries, 29(3), $178-194$.

Xie, H. (2000). Shifts of interactive intentions and information-seeking strategies in interactive information retrieval. Journal of the American Society for Information Science, 51(9), 841-857.

Vakkari, P. (1999). Task complexity, problem structure and information actions, integrated studies on information seeking and retrieval. Information Processing and Management, 35, 819-837.

Vakkari, P. (2001). Changes in search tactics and relevance judgments when preparing a research proposal: a summary and generalization of a longitudinal study. Journal of Documentation, 57(1), 44-60.

Wilson, T. (1999). Exploring models of information behavior: the 'uncertainty' project. Information Processing and Management, $35,839-849$.

Wilson, T. (1999). Models in information behavior research. The Journal of Documentation, 55(3), $249-270$.

Wilson, T.D., Ford, N., Foster, A., Spink, A. (2002). Information seeking and mediated searching. Part 2. Uncertainty and its correlates. Journal of the American Society for Information Science, 53(9), 704-715. 